\title{
Original Research \\ Bleeding risk in patients with atrial fibrillation treated with combined anti-platelet and non-vitamin $K$ antagonist oral anticoagulant therapy
}

\author{
Dong Geum Shin ${ }^{1}$, Sunhwa Kim², Yoo Ri Kim,* \\ ${ }^{1}$ Division of Cardiology, Department of Internal medicine, Hallym University Kangnam Sacred Heart Hospital, 07226 Seoul, Republic of Korea \\ ${ }^{2}$ Division of Cardiology, The Catholic University of Korea, Incheon St. Mary's Hospital, 21431 Incheon, Republic of Korea \\ ${ }^{3}$ Division of Cardiology, Department of Internal medicine, Dongguk University College of Medicine, Ilsan Hospital, Goyang-si, 10326 Gyeonggi-do, \\ Republic of Korea \\ *Correspondence: yul@dumc.or.kr (Yoo Ri Kim) \\ Academic Editors: Giuseppe Nasso and Giuseppe Santarpino \\ Submitted: 24 August 2021 Revised: 8 October 2021 Accepted: 27 October 2021 Published: 7 January 2022
}

\begin{abstract}
Background: The use of non-vitamin K antagonist oral anticoagulants (NOACs) in patients with non-valvular atrial fibrillation (AF) has been increasing. Accordingly, the combined use of antiplatelet agents (APT) and NOAC therapy is commonly encountered in clinical practice. The purpose of this study was to compare the clinical outcomes between combination therapy (NOAC and APT) vs. monotherapy (NOAC only) in patients with AF. Methods: We retrospectively analyzed patients who were prescribed NOACs between January 2012 and December 2016. The primary outcome was major bleeding and any bleeding events, and the secondary outcomes were stroke/systemic embolic (SE) events and major adverse cardiac events (MACE). Results: Of the 1068 participants, there were 264 (24.7\%) patients in the combination therapy group. The prevalence of diabetes $(p=0.017)$ and history of stroke and transient ischemic attacks $(p<0.001)$ was higher in the combination group than in the monotherapy group. During the mean $14.6 \pm 9.8$ months of followup, the incidence of any bleeding was significantly higher in the combination therapy group than in the monotherapy group $(p<0.001)$. The rate of major bleeding, stroke/SE, and MACE between the two groups was similar. The rate of under-dosage NOAC prescriptions was higher in the combination therapy group than in the monotherapy group $(p=0.024)$. Conclusions: The combination therapy group had higher incidences of any bleeding events compared to the monotherapy in patients with appropriate dosing. However, there was no difference in stroke/SE, and MACE. The bleeding risk in AF patients taking the combination of NOACs and APT should be carefully evaluated.
\end{abstract}

Keywords: Antiplatelet agents; Atrial fibrillation; Bleeding; Non-vitamin K oral anticoagulant

\section{Introduction}

Atrial fibrillation (AF) is the most common form of sustained cardiac arrhythmia. According to the guidelines, it is estimated that $15 \%$ of strokes occur in patients with AF [1]. Thus, oral anticoagulants (OACs) have been the cornerstone of stroke and systemic embolism (SE) prophylaxis in patients with AF. Several studies including both randomized controlled trials and real world-settings have shown that non-vitamin $\mathrm{K}$ antagonist oral anticoagulants (NOACs) in patients with non-valvular AF were more effective than warfarin in preventing thromboembolic events and reducing the risk of bleeding events [2-6]. Therefore, NOACs are preferred over warfarin, and NOAC prescriptions have increased rapidly [7].

Most patients with AF also have various risk factors for atherosclerotic cardiovascular diseases [8]. Therefore, the proper strategy of combination of OACs and antiplatelet therapy (APT) in many conditions has great clinical impact. However, current strategies combining OACs and APT therapies have led to a significant increase in bleeding rates $[9,10]$. Studies have reported conflicting results regarding the combination of OACs and APT to optimize ischemia and bleeding risk. For this reason, finding the optimal balance of the appropriate treatments for each patient with indicators for both treatments is critical in achieving clinical benefits.

Compared to prescribing adequate warfarin doses according to the prothrombin time, each NOAC has a recommended dose based on the clinical characteristics of the patient, and criteria are provided for dose reduction. Although NOAC prescriptions have increased gradually, the number of patients receiving an under-dosing NOACs for various reasons has also increased $[11,12]$. However, patients with AF who are receiving suboptimal medical therapy are at an increased risk of stroke, cardiovascular hospitalization, and mortality [13].

The main objective of this retrospective study was to evaluate the clinical safety and efficacy of combination therapy (NOAC and APT) compared to monotherapy (NOAC only) in a real-world cohort of AF patients. In addition, we aimed to investigate whether the under-dosing of NOACs was effective or safe. 


\section{Methods}

\subsection{Study population}

We retrospectively analyzed 1068 consecutive patients who were prescribed initiating NOACs for AF at Catholic University St. Mary's Hospital, in Incheon, Korea between January 2012 and December 2016. The diagnosis of AF was based on a 12-lead electrocardiogram characterized by the absence of discrete P-waves and an irregular ventricular rate. Paroxysmal AF (PAF) was defined in patients with a history of $\geq 1$ episode of self-terminating $\mathrm{AF}$ that lasted $\leq 7$ days. The inclusion criteria were nonvalvular AF patients who were taking NOACs and some of them were taking APT. The exclusion criteria were patients less than 18 years old, with a history of NOAC therapy, mechanical prosthetic valves or moderate-to-severe mitral stenosis, and impaired renal function [creatinine clearance $(\mathrm{CrCl})<30 \mathrm{~mL} / \mathrm{min}$ and dialysis]. We also excluded patients with coronary or peripheral artery disease undergoing stent implantation within the last 12 months. The patients were divided into combination therapy or monotherapy groups according to whether or not APT was administered with NOACs. All case records were reviewed following a standardized data collection protocol for the purpose of obtaining information. The authors had full access to and take full responsibility for the integrity of the data. The study protocol adhered to the principles of the Declaration of Helsinki and was approved by the Institutional Review Board.

\subsection{Antiplatelet agents and NOACs}

The types and doses of APT administered included aspirin, triflusal, P2Y12 inhibitors, or cilostazol taken for various reasons or no apparent reason. There were three types of NOACs administered, apixaban, dabigatran, and rivaroxaban. A standard dose was defined as apixaban at $5 \mathrm{mg}$ twice daily, dabigatran at $150 \mathrm{mg}$ twice daily, and rivaroxaban at $20 \mathrm{mg}$ once daily. A dose reduction protocol was based on the 2018 European Heart Rhythm Association practical guideline [14]. Dabigatran was administered at $110 \mathrm{mg}$ twice a day to patients with moderate renal impairment ( $\mathrm{CrCl} 15-29 \mathrm{~mL} / \mathrm{min})$, those who had been taking APT or non-steroidal anti-inflammatory drugs, or patients 75 years of age or older. If in patients over 75 -year-old or a $\mathrm{CrCl}$ was $30-50 \mathrm{~mL} / \mathrm{min}, 15 \mathrm{mg}$ of rivaroxaban was given once a day. Apixaban was administered at $2.5 \mathrm{mg}$ twice a day for two or more of the following three conditions: patients over 80 years old, a weight of less than $60 \mathrm{~kg}$, and a serum $\mathrm{Cr}$ value of less than $1.5 \mathrm{mg} / \mathrm{dL}$. When the dose was prescribed according to the above criteria, it was defined as appropriate dosing. The dosage of APT given with NOACs was prescribed according to the physician's judgment.

\subsection{Clinical outcomes}

The primary outcome was major bleeding and any bleeding. Major bleeding was defined as clinically overt bleeding requiring hospital admission including a blood transfusion, decreased hemoglobin levels of $\geq 2.0 \mathrm{~g} / \mathrm{dL}$, or symptomatic bleeding in a critical area or organ. The secondary outcomes were stroke or SE, and major adverse cardiac events (MACE). Definite stroke or SE was diagnosed by the combination of both the clinical situation and radiologic studies. MACE was a composite of bleeding events, thromboembolic events, and deaths including cardiovascular, non-cardiovascular, and undetermined deaths.

\subsection{Statistical analysis}

The continuous variables are presented as means and standard deviations. Comparison of the continuous variables was performed using an independent $t$-test or, in case of a non-normal distribution, the Mann-Whitney test. The categorical variables are reported as counts and proportions and analyzed using Pearson's Chi-squared tests or Fisher's exact test, as appropriate. Kaplan-Meier analysis with the log-rank test was used to compare the clinical outcomes. Multivariate Cox proportional regression analysis was used to investigate any independent predictors of clinical outcomes. The SPSS statistical package (SSPS Inc., Chicago, IL, USA) was used to perform all statistical evaluations. A $p$-value of $<0.05$ was considered statistically significant.

\section{Results}

\subsection{Baseline characteristics}

The study population consisted of 1068 patients with AF taking NOACs. Of these, 804 patients $(75.3 \%)$ were taking monotherapy, and 264 (24.7\%) were taking combination therapy. Table 1 shows a summary of the baseline characteristics of the overall study population according to the use of APT. The mean patient age was $70.5 \pm 12.1$ years, and $528(49.4 \%)$ were male. The mean $\mathrm{CHA}_{2} \mathrm{DS}_{2}$ VASc score was $3.1 \pm 1.6$, and $538(50.4 \%)$ patients had PAF.

Compared to the monotherapy group, the prevalence of diabetes $(25.4 \%$ vs. $18.6 \%, p=0.017)$, stroke/TIA history $(44.7 \%$ vs. $22.9 \%, p<0.001)$ and peripheral vascular disease $(0.4 \%$ vs. $2.3 \%, p=0.009)$ were significantly higher in the combination therapy group. Other clinical characteristics including heart failure, hypertension, myocardial infarction and CHA2DS2-VASc scores demonstrated no significant difference between the groups.

\subsection{Clinical outcomes}

During $14.6 \pm 9.8$ months of follow-up, 37 (3.5\%) patients had major bleeding, $86(8.1 \%)$ patients had any bleeding, 47 (4.4\%) patients had stroke or SE, and 45 (4.2\%) patients died from cardiac or non-cardiac causes. The patients taking combination therapy had a higher rate of any bleeding $(15.2 \%$ vs. $5.7 \% ; p<0.001)$ and similar rates of major bleeding, stroke or SE, and MACE (all $p>0.05)$. The types of thromboembolic and bleeding events reported in the two groups are listed in Table 2. 
Table 1. Baseline characteristics of patients taking monotherapy or combination therapy.

\begin{tabular}{lcccc}
\hline & Overall & Monotherapy & Combination therapy & \multirow{2}{*}{$p$-value } \\
\cline { 2 - 4 } & $(\mathrm{n}=1068)$ & $(\mathrm{n}=804)$ & $(\mathrm{n}=264)$ & \\
\hline Age & $70.5 \pm 12.1$ & $70.4 \pm 12.5$ & $70.6 \pm 11.0$ & 0.804 \\
Sex, male, \% & $528(49.4)$ & $384(47.6)$ & $145(54.9)$ & 0.040 \\
Body mass index, $\mathrm{kg} / \mathrm{m}^{2}$ & $24.5 \pm 3.9$ & $24.4 \pm 4.0$ & $24.8 \pm 3.8$ & 0.208 \\
Creatinine Clearance, $\mathrm{mL} / \mathrm{min}$ & $62.9 \pm 27.7$ & $62.7 \pm 28.0$ & $63.4 \pm 26.7$ & 0.454 \\
Paroxysmal AF, \% & $538(50.4)$ & $393(48.9)$ & $145(54.9)$ & 0.088 \\
Previous warfarin use & $49(4.6)$ & $32(4.0)$ & $17(6.4)$ & 0.098 \\
Medical history, \% & & & & \\
$\quad$ Heart failure & $135(12.6)$ & $99(12.3)$ & $36(12.3)$ & 0.575 \\
$\quad$ Hypertension & $482(45.3)$ & $361(45.1)$ & $121(45.8)$ & 0.829 \\
$\quad$ Diabetes mellitus & $216(20.2)$ & $149(18.6)$ & $67(25.4)$ & 0.017 \\
$\quad$ Stroke/TIA & $302(28.3)$ & $184(22.9)$ & $118(44.7)$ & $<0.001$ \\
$\quad$ Myocardial infarction & $29(2.7)$ & $21(2.6)$ & $8(3.0)$ & 0.717 \\
$\quad$ Peripheral vascular disease & $9(3.6)$ & $3(0.4)$ & $6(2.3)$ & 0.009 \\
$\quad$ CHA ${ }_{2}$ DS ${ }_{2}$-VASc score & $3.1 \pm 1.6$ & $2.9 \pm 1.6$ & $3.4 \pm 1.7$ & 0.493 \\
Echocardiographic value & & & & 0.789 \\
$\quad$ LV ejection fraction, \% & $58.1 \pm 10.1$ & $58.1 \pm 10.2$ & $27.9 \pm 9.5$ & 0.065 \\
$\quad$ LA size (AP diameter), mm & $43.8 \pm 7.7$ & $43.5 \pm 7.6$ & $44.5 \pm 7.8$ & \\
\hline
\end{tabular}

$\mathrm{AF}$, atrial fibrillation; BMI, body mass index; $\mathrm{CHA}_{2} \mathrm{DS}_{2}$-VASc, Congestive heart failure, Hypertension, Age $\geq 75$ (doubled), Diabetes mellitus, and prior ischemic Stroke, transient ischemic attack or thromboembolism (doubled), Vascular disease, Age 65 to 74, Sex category (female); LA, left atrium; TIA, transient ischemic attack.

As shown in Fig. 1, the Kaplan-Meier analysis showed a significant difference in event-free survival from any bleeding (log-rank $p=0.018$ ). However, the incidence of major bleeding (log-rank $p=0.149)$, stroke or SE (log-rank $p=0.885)$, and MACE (log-rank $p=0.916)$ was not different between the two groups. As shown in in the Supplementary Tables, Combination therapy was associated with major bleeding on multivariate Cox proportional hazard analysis (Hazard ratio [HR], 2.74; 95\% confidence interval [Cl]: 1.01-7.44). And old age was independent risk factor of MACE.

\subsection{Preferred dose of NOAC}

Table 3 shows a comparison according to the dose of NOAC. Among the entire study population, the number of patients receiving appropriate dosing was 540 (50.6\%). There were 482 (45.1\%) under-dosed patients, and 45 (4.2\%) received over-dosages. Treatment preference was found to depend upon concomitant APT. The combination group had a lower proportion of appropriate dosage prescriptions and a higher proportion of under-dosage prescriptions than the monotherapy group.

The proportions of appropriate, under-dosage, and over-dosage prescriptions differed among NOACs. Most patients taking dabigatran and apixaban received appropriate dosage prescriptions, whereas rivaroxaban showed a higher proportion of under-dosage prescriptions. In the case of rivaroxaban, under-dosage prescriptions were administered more in the combination group.

\subsection{Clinical outcomes according to the dose of NOAC}

In sub-analysis based on appropriately prescribed doses of NOACs, depending upon whether APT was taken or not, all outcomes were similar to those seen in the overall population (major bleeding: $\log$-rank $p=0.722$, any bleeding: $\log$-rank $p=0.001$, stroke or SE: $\log$-rank $p=0.744$, and MACE: $\log$-rank $p=0.237$; Fig. 2).

The clinical outcomes of patients prescribed appropriate monotherapy doses and under-dosages in the combination group were compared. In the Kaplan-Meier analysis, under-dosing in the combination group showed no significant difference in the event-free survival of patients with major bleeding (log-rank $p=0.874)$, any bleeding (log-rank $p=0.810)$, and MACE (log-rank $p=0.864)$ but increased stroke and SE rates (log-rank $p=0.001)$ compared to patients in the appropriate dosage monotherapy group (Fig. 3).

\section{Discussion}

The major findings of this real-world retrospective study were that combination therapy with NOACs plus APT was (1) not uncommon, (2) increased any bleeding events but did not decrease stroke/SE events, and (3) under-dosage prescriptions of NOAC occurred at a high rate. 
Table 2. Reported thromboembolic and bleeding events.

\begin{tabular}{|c|c|c|c|c|}
\hline & Overall & Monotherapy & Combination therapy & \multirow{2}{*}{$p$-value } \\
\hline & $(\mathrm{n}=1068)$ & $(n=804)$ & $(n=264)$ & \\
\hline Thromboembolic event (\%) & $47(4.4)$ & $31(3.9)$ & $16(6.1)$ & 0.130 \\
\hline Stroke & 44 & 28 & 16 & \\
\hline Systemic embolism & 3 & 3 & 0 & \\
\hline Any bleeding event (\%) & $86(8.1)$ & $46(5.7)$ & $40(15.2)$ & $<0.001$ \\
\hline Major bleeding (\%) & $37(3.5)$ & $29(3.6)$ & $8(3.0)$ & 0.657 \\
\hline Gastro-intestinal & 23 & 19 & 4 & \\
\hline Intracranial & 7 & 4 & 3 & \\
\hline Hemoptysis & 5 & 4 & 1 & \\
\hline Pericardial effusion & 1 & 1 & 0 & \\
\hline Aortic rupture & 1 & 1 & 0 & \\
\hline Non-Major bleeding (\%) & $49(4.6)$ & $17(2.1)$ & $32(12.1)$ & $<0.001$ \\
\hline Cutaneous & 22 & 8 & 14 & \\
\hline Epistaxis & 12 & 4 & 8 & \\
\hline Hematuria & 9 & 3 & 6 & \\
\hline Oral cavity & 6 & 2 & 4 & \\
\hline
\end{tabular}

\section{A. Major bleeding}

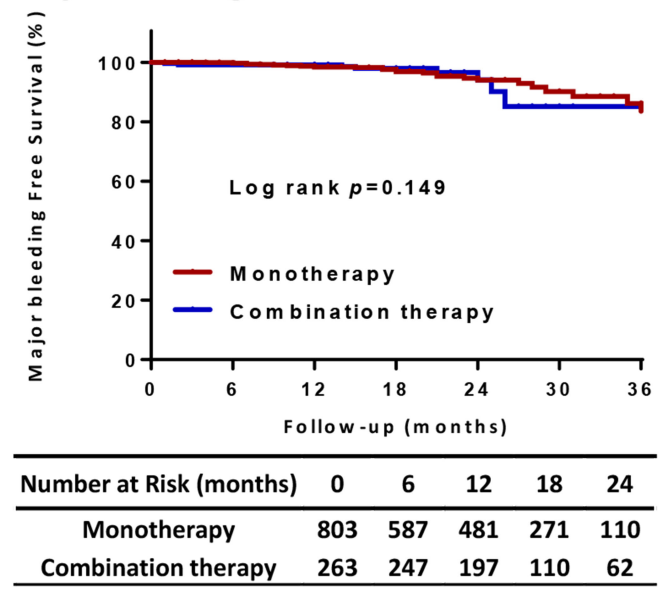

C. Stroke or SE

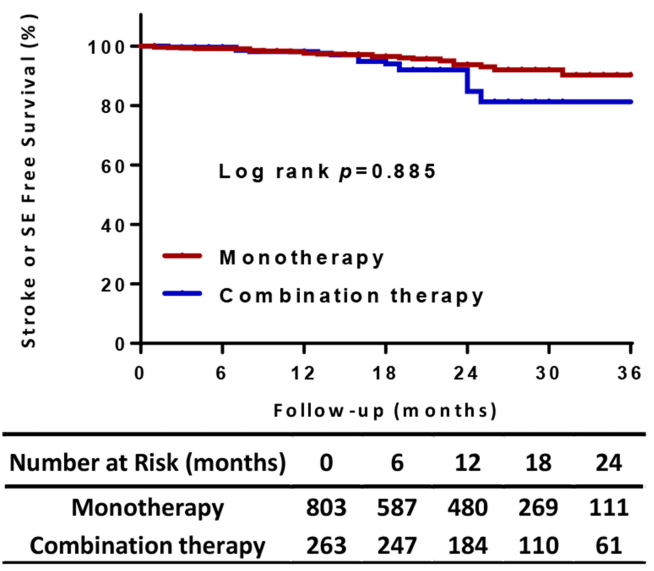

B. Any bleeding

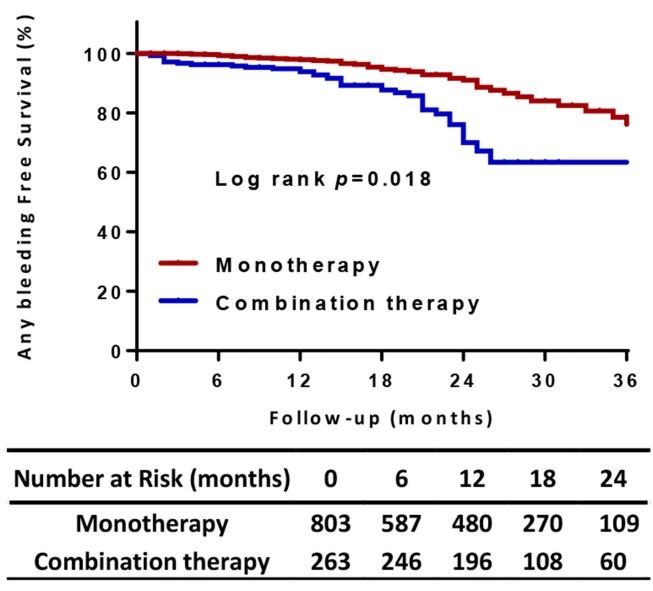

D. MACE

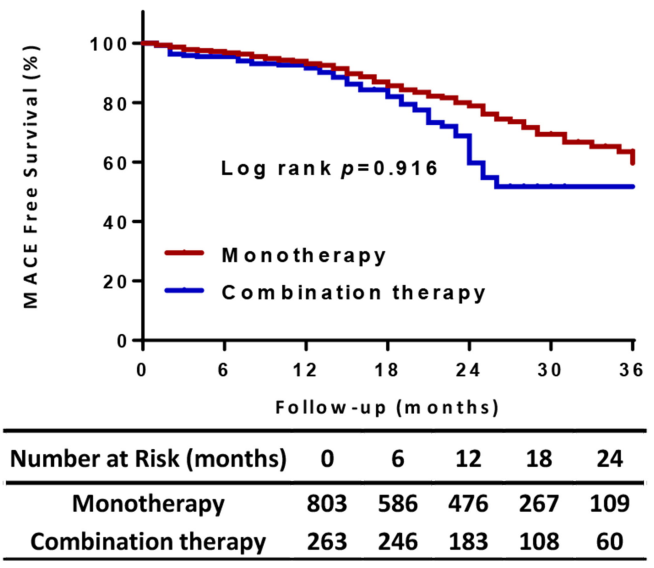

Fig. 1. Kaplan-Meier curves for the incidences of (A) major bleeding, (B) any bleeding (C) stroke or systemic embolism (SE), and (D) major adverse cardiac events (MACE) of entire study population according to monotherapy (NOAC only) or combination therapy (NOAC and APT). 
Table 3. Comparison according to the dose of NOACs.

\begin{tabular}{lcccc}
\hline & Overall & Monotherapy & Combination therapy & \multirow{2}{*}{$p$-value } \\
\cline { 2 - 4 } & $(\mathrm{n}=632)$ & $(\mathrm{n}=141)$ & $(\mathrm{n}=491)$ & \\
\hline Over-dosage & $43(4.0)$ & $35(4.4)$ & $8(3.0)$ & 0.343 \\
Appropriate dosage & $530(49.6)$ & $422(52.5)$ & $108(40.9)$ & 0.001 \\
Under-dosage & $482(45.1)$ & $347(43.2)$ & $135(51.1)$ & 0.024 \\
Dabigatran & $227(21.3)$ & $137(17.0)$ & $90(34.1)$ & \\
$\quad 11(4.8)$ & $8(5.8)$ & $3(3.3)$ & 0.390 \\
Over-dosage & $143(63.0)$ & $96(70.1)$ & $47(52.2)$ & 0.006 \\
Appropriate dosage & $64(28.2)$ & $33(24.1)$ & $31(34.4)$ & 0.090 \\
Under-dosage & $380(35.6)$ & $290(36.1)$ & $90(34.1)$ & \\
Rivaroxaban & $19(5.0)$ & $16(5.5)$ & $3(3.3)$ & 0.406 \\
$\quad$ Over-dosage & $123(32.4)$ & $101(34.8)$ & $22(24.4)$ & 0.066 \\
Appropriate dosage & $238(62.6)$ & $173(59.7)$ & $65(72.2)$ & 0.031 \\
Under-dosage & $461(43.2)$ & $377(46.9)$ & $84(31.8)$ & \\
Apixaban & $13(2.8)$ & $11(2.9)$ & $2(2.4)$ & 0.788 \\
$\quad$ Over-dosage & $264(57.3)$ & $225(59.7)$ & $39(46.4)$ & 0.026 \\
Appropriate dosage & $180(39.0)$ & $141(37.4)$ & $39(46.4)$ & 0.125 \\
$\quad$ Under-dosage & 18.4 & & \\
\hline
\end{tabular}

NOAC, non-vitamin K oral anticoagulants.

\section{A. Major bleeding}

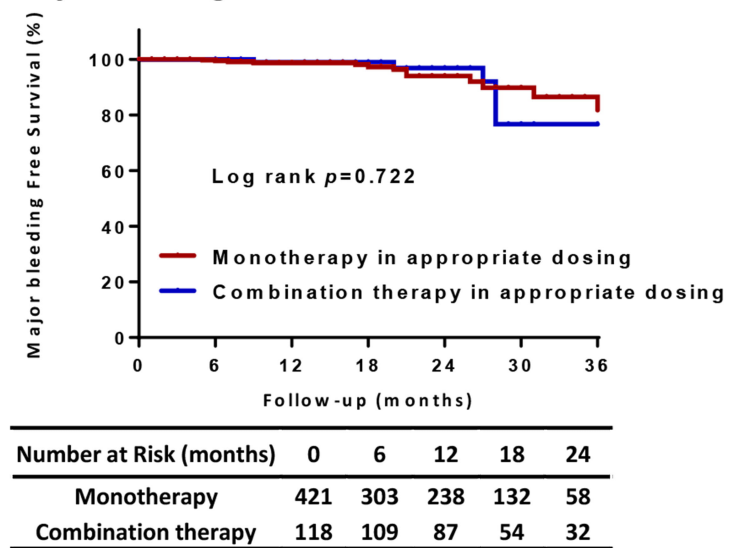

\section{Stroke or SE}

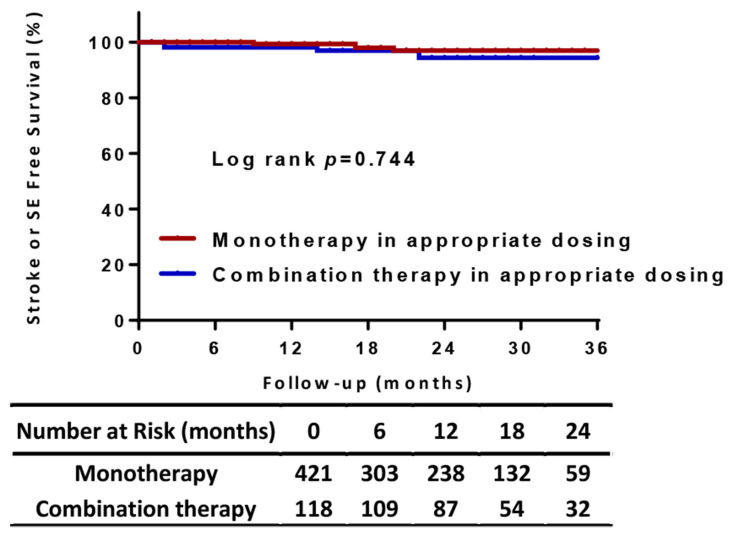

\section{B. Any bleeding}

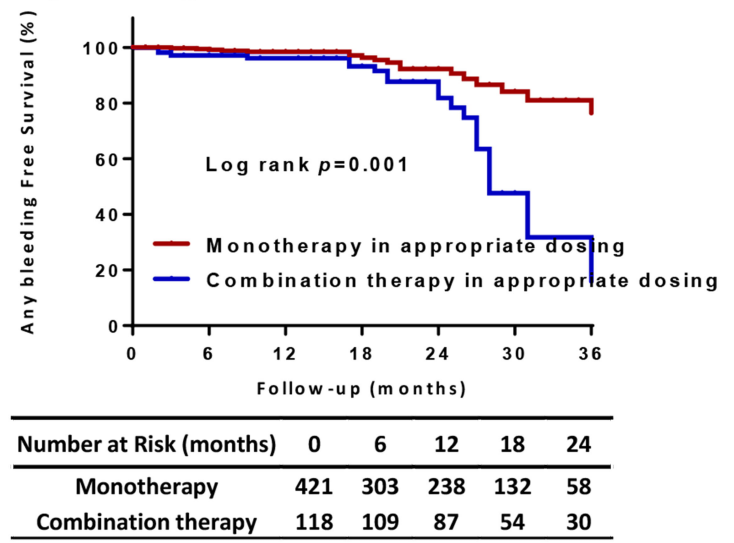

D. MACE

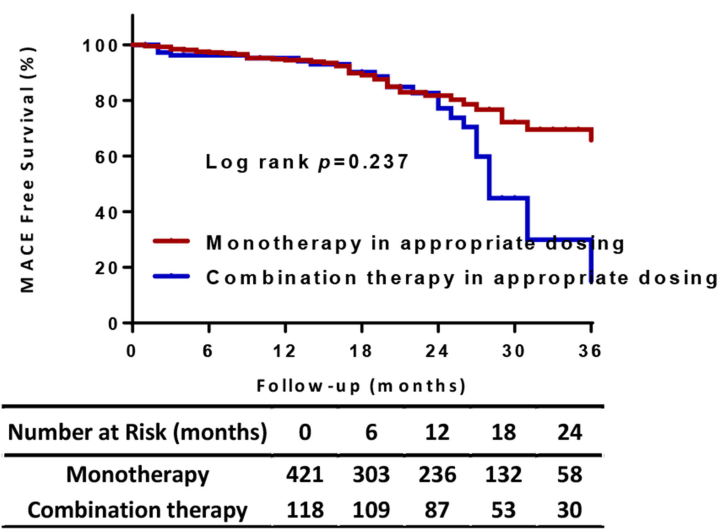

Fig. 2. Kaplan-Meier curves for the incidences of (A) major bleeding, (B) any bleeding (C) stroke or systemic embolism (SE), and (D) major adverse cardiac events (MACE) of patients with appropriate dosing according to monotherapy (NOAC only) or combination therapy (NOAC and APT). 
A. Major bleeding

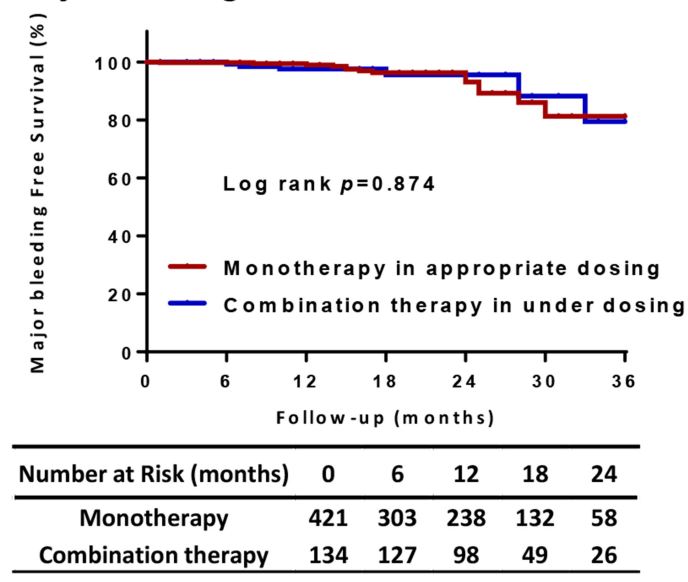

C. Stroke or SE

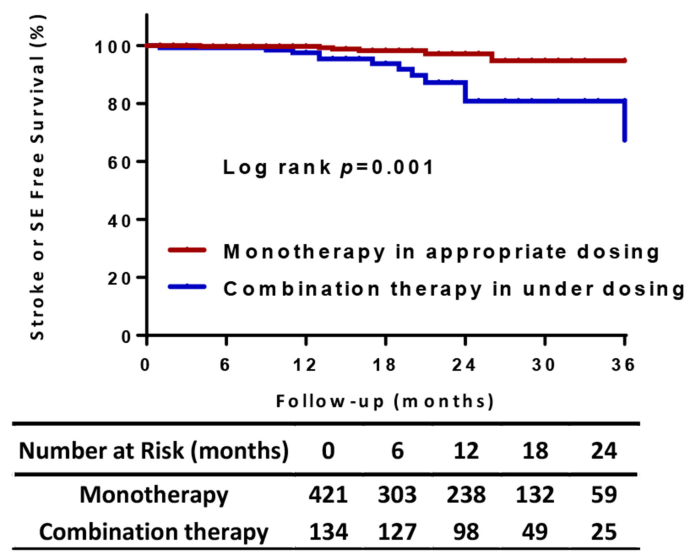

B. Any bleeding

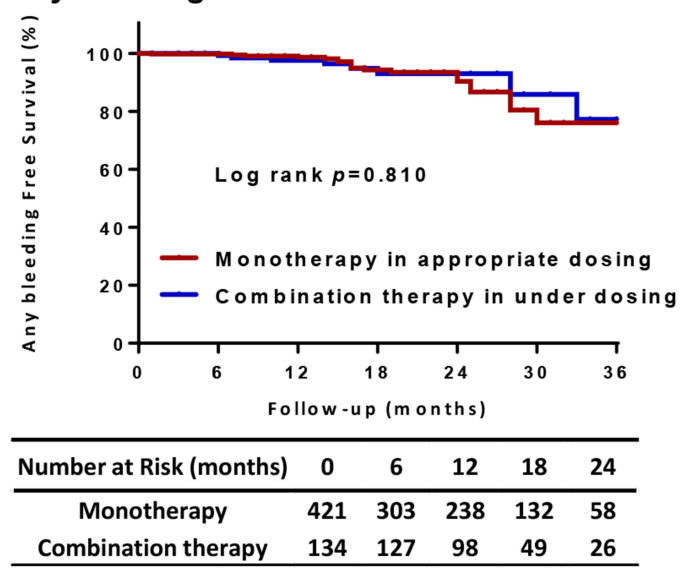

D. MACE

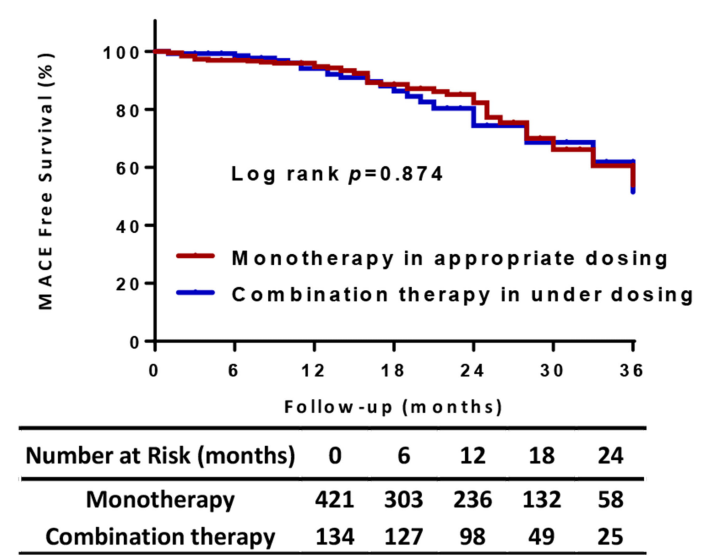

Fig. 3. Kaplan-Meier curves for the incidences of (A) major bleeding, (B) any bleeding (C) stroke or systemic embolism (SE), and (D) major adverse cardiac events (MACE) of some patients according to appropriate dosing monotherapy (NOAC only) or under dosing combination therapy (NOAC and APT).

Numerous studies have demonstrated that APT in patients with AF was inferior or not superior to OACs in terms of bleeding risk or benefit in stroke prevention [15]. Therefore, APT is no longer recommended as a treatment for stroke prevention in patients with AF $[16,17]$. However, many patients with AF have risk factors for cardiovascular events [8]. So APT is often administered in combination with NOACs in clinical settings. Combination therapy was administrated to $25.4 \%$ of the patients in this study. This was similar to other real-world registries $[18,19]$ and randomized trials [20-23]. However, contrary to the results of this study, Ruiz et al. [24] reported that the use of combination therapy in AF patients initiating NOAC treatment was uncommon, at $6.1 \%$. And $12.5 \%$ of the patients were treated with APT in addition to OACs in the GARFIELDAF registry [25]. This finding was explained by the baseline characteristics of the enrolled patients. The study by Ruiz et al. [24] excluded patients with established indications for concomitant APT and the GARFIELD-AF registry included patients who had taken vitamin $\mathrm{K}$ antagonist.
In our study, patients taking combination therapy had higher bleeding event rates and no significant difference in stroke/SE events and MACE, consistent with previous findings. The combination of OACs and APT drugs increases the bleeding potential. Meta-analysis and observational studies demonstrated an increased risk of bleeding and no additional benefit for stroke prevention in patients given combination therapy [18,19,24,26]. Patients with combination therapy had higher incidences of major bleeding as well as stroke/SE, compared to those given monotherapy in the Fushimi AF Registry [11]. In a subanalysis of the GARFIELD-AF registry [25], the patients treated with OACs plus APT experienced a higher incidence of adverse outcomes during the observation period. In another study similar to our study, the use of concomitant APT was not associated with lower rates of ischemic events or death, whereas there was an increased risk of bleeding. Although several studies demonstrated that APT for the secondary prevention of cardiovascular events improved clinical outcomes [27,28], the additional efficacy of APT was not demonstrated and less clear in AF patients receiving 
OACs. Also, combination therapy has been mainly investigated in patients with AF who had undergone percutaneous coronary intervention (PCI). In the AFIRE trial [29], as in our study, patients with remote ( $>12$ months) history of PCI who treated with NOAC monotherapy group demonstrated superior for safety than the combination (NOAC plus APT) therapy, and the study was terminated early. Additionally, a recent review article on antithrombotic therapy after PCI in Asians with AF reported that OAC monotherapy is reasonable if it exceeds 1 year [30]. These reports could support our findings. Our study could provide better assistance in decision making in the real practice.

In this study, approximately $45 \%$ of the patients were prescribed an under-dosage of NOAC. In the USA, $83.7 \%$ of the patients were prescribed the recommended dosage of NOACs, whereas $12.0 \%$ of the patients received an underdosage of NOACs [31]. In the Korean National Health Insurance Service database, $51.9 \%$ of the patients were prescribed an under-dosage of NOAC [12]. Additionally, In Japan, $50 \%$ of the patients receiving reduced-dose NOACs were prescribed an under-dosage of NOAC [32]. Underdosing with NOACs in Asians was more common than in Westerners. Physicians tend to be more anxious about the side effects of bleeding than the risk of stroke [33]. In particular, Asians have a small body size and are known to have a high frequency of cerebral hemorrhages and gastrointestinal bleeding associated with the use of OACs, so physicians in Asia tend to administer an under-dosage of NOACs [34]. Also, concomitant APT use was associated with under-dosage NOAC prescriptions. According to observational studies [35], the combination group had a higher proportion of under-dosage prescriptions than the monotherapy group, consistent with our findings. Recently, adverse clinical outcomes in patients prescribed under-dosages of NOACs have been reported from real-world data [12]. In the ORBIT-AF II registry, compared to the patients appropriately dosed with NOACs, patients given under-dosages of NOAC showed higher adverse event rates [13]. Our results demonstrated that under-dosed patients in the combination group showed increased stroke/SE events compared to appropriately dosed patients in the monotherapy group. The appropriate use of NOACs is emerging as an important issue.

There were several limitations to this study. First, the data in the study were acquired from electronic medical records, retrospectively. Due to the limitation of a retrospective study, it is possible that the clinical results were measured lower than the actual incidence rate. However, this would not have affected the comparison of the results in the monotherapy group and the combination group. Second, the usage of APT was based on each physicians' preference and the patient's baseline characteristics and it also might affect the physician's choice of the dosage of NOACs by the previous history of bleeding or thromboembolic events. It is an inherent limitation of retrospective re- search. The bias was already included in the study design. The baseline characteristics and all clinical outcomes were made only through the medical records. There was a lack of information on lifestyle, social habits: drinking and smoking, or using concomitant drugs, such as NSAIDs. Finally, this was a single-center retrospective study with relatively small number of the patients with combination therapy and the variable baseline characteristics in both groups. The results cannot be generalized, and a further prospective study was needed to confirm the safety for the combination therapy.

\section{Conclusions}

Patients taking combination therapy had higher incidences of any bleeding compared to AF patients taking monotherapy, whereas no significant difference was seen in stroke prevention. Many patients were prescribed underdosages of NOAC, especially in the combination therapy group. Careful evaluation of the indications for APT in patients with AF who are also taking NOACs is warranted, and a future randomized comparison study is needed.

\section{Abbreviations}

$\mathrm{AF}$, atrial fibrillation; APT, antiplatelet agents; $\mathrm{CrCl}$, creatinine clearance; MACE, major adverse cardiac events; $\mathrm{HR}$, hazard ratio; NOACs, non-vitamin $\mathrm{K}$ antagonist oral anticoagulants; PAF, paroxysmal AF; PCI, percutaneous coronary intervention; SE, systemic embolic; TIA, transient ischemic attack.

\section{Author contributions}

All authors contributed and participated in the preparation of the manuscript and research steps in the present study as follows: Conception and Design and Supervision-YRK. Acquisition of data-SK, YRK. Analysis of data and Writing of original draft_-DGS. Reading, Revising and Final approval for submission-All.

\section{Ethics approval and consent to participate}

This study protocol was approved by Incheon St. Mary's Hospital Institutional Review Board/2020-40260001 .

\section{Acknowledgment}

Not applicable.

\section{Funding}

This research received no external funding.

\section{Conflict of interest}

The authors declare no conflict of interest. 


\section{Supplementary material}

Supplementary material associated with this article can be found, in the online version, at https://www.imrpre ss.com/journal/RCM/23/1/10.31083/j.rcm2301002.

\section{References}

[1] Lip GY, Lim HS. Atrial fibrillation and stroke prevention. The Lancet Neurology. 2007; 6: 981-993.

[2] Connolly SJ, Ezekowitz MD, Yusuf S, Eikelboom J, Oldgren $\mathrm{J}$, Parekh A, et al. Dabigatran versus warfarin in patients with atrial fibrillation. New England Journal of Medicine. 2009; 361: $1139 \neg-1151$.

[3] Patel MR, Mahaffey KW, Garg J, Pan G, Singer DE, Hacke W, et al. Rivaroxaban versus Warfarin in Nonvalvular Atrial Fibrillation. New England Journal of Medicine. 2011; 365: 883-891.

[4] Granger CB, Alexander JH, McMurray JJ, Lopes RD, Hylek $\mathrm{EM}$, Hanna M, et al. Apixaban versus warfarin in patients with atrial fibrillation. New England Journal of Medicine. 2011; 365 : 981-992.

[5] Giugliano RP, Ruff CT, Braunwald E, Murphy SA, Wiviott SD, Halperin JL, et al. Edoxaban versus warfarin in patients with atrial fibrillation. The New England Journal of Medicine. 2013; 369: 2093-2104.

[6] Ntaios G, Papavasileiou V, Makaritsis K, Vemmos K, Michel P, Lip GYH. Real-World Setting Comparison of Nonvitamin-K Antagonist Oral Anticoagulants Versus Vitamin-K Antagonists for Stroke Prevention in Atrial Fibrillation: a Systematic Review and Meta-Analysis. Stroke. 2017; 48: 2494-2503.

[7] Huisman MV, Rothman KJ, Paquette M, Teutsch C, Diener H, Dubner SJ, et al. The Changing Landscape for Stroke Prevention in AF: Findings from the GLORIA-AF Registry Phase 2. Journal of the American College of Cardiology. 2017; 69: 777-785.

[8] Verheugt FWA, Ambrosio G, Atar D, Bassand JP, Camm AJ, Costabel JP, et al. Outcomes in Newly Diagnosed Atrial Fibrillation and History of Acute Coronary Syndromes: Insights from GARFIELD-AF. The American Journal of Medicine. 2019; 132: 1431-1440.e7.

[9] Sørensen R, Hansen ML, Abildstrom SZ, Hvelplund A, Andersson C, Jørgensen C, et al. Risk of bleeding in patients with acute myocardial infarction treated with different combinations of aspirin, clopidogrel, and vitamin $\mathrm{K}$ antagonists in Denmark: a retrospective analysis of nationwide registry data. The Lancet. 2009; 374: 1967-1974.

[10] Lamberts M, Olesen JB, Ruwald MH, Hansen CM, Karasoy D, Kristensen SL, et al. Bleeding after initiation of multiple antithrombotic drugs, including triple therapy, in atrial fibrillation patients following myocardial infarction and coronary intervention: a nationwide cohort study. Circulation. 2012; 126: 11851193.

[11] Masunaga N, Abe M, Ogawa H, Aono Y, Ikeda S, Doi K, et al. Current Status, Time Trends and Outcomes of Combination Therapy with Oral Anticoagulant and Antiplatelet Drug in Patients with Atrial Fibrillation $\neg\urcorner$ - The Fushimi AF Registry. Circulation Journal. 2018; 82: 2983-2991.

[12] Cho MS, Yun JE, Park JJ, Kim YJ, Lee J, Kim H, et al. Pattern and Impact of off-label Underdosing of Non-Vitamin K Antagonist Oral Anticoagulants in Patients with Atrial Fibrillation who are Indicated for Standard Dosing. The American Journal of Cardiology. 2020; 125: 1332-1338.

[13] Steinberg BA, Shrader P, Thomas L, Ansell J, Fonarow GC, Gersh BJ, et al. Off-Label Dosing of Non-Vitamin K Antagonist Oral Anticoagulants and Adverse Outcomes: the ORBIT-AF II Registry. Journal of the American College of Cardiology. 2016; 68: 2597-2604.

[14] Steffel J, Verhamme P, Potpara TS, Albaladejo P, Antz M,
Desteghe L, et al. The 2018 European Heart Rhythm Association Practical Guide on the use of non-vitamin K antagonist oral anticoagulants in patients with atrial fibrillation. European Heart Journal. 2018; 39: 1330-1393.

[15] Warfarin versus aspirin for prevention of thromboembolism in atrial fibrillation: Stroke Prevention in Atrial Fibrillation II Study. The Lancet. 1994; 343: 687-691.

[16] January CT, Wann LS, Calkins H, Chen LY, Cigarroa JE, Cleveland JC Jr., et al. 2019 AHA/ACC/HRS Focused Update of the 2014 AHA/ACC/HRS Guideline for the Management of Patients with Atrial Fibrillation: A Report of the American College of Cardiology/American Heart Association Task Force on Clinical Practice Guidelines and the Heart Rhythm Society in Collaboration with the Society of Thoracic Surgeons. Circulation. 2019; 140: e125-e151.

[17] Hindricks G, Potpara T, Dagres N, Arbelo E, Bax JJ, Blomstrom-Lundqvist C, et al. 2020 ESC Guidelines for the diagnosis and management of atrial fibrillation developed in collaboration with the European Association of Cardio-Thoracic Surgery (EACTS): The Task Force for the diagnosis and management of atrial fibrillation of the European Society of Cardiology (ESC) Developed with the special contribution of the European Heart Rhythm Association (EHRA) of the ESC. European Heart Journal. 2021; 42: 373-498.

[18] Lamberts M, Gislason GH, Lip GYH, Lassen JF, Olesen JB, Mikkelsen AP, et al. Antiplatelet therapy for stable coronary artery disease in atrial fibrillation patients taking an oral anticoagulant: a nationwide cohort study. Circulation. 2014; 129: $1577-1585$.

[19] Steinberg BA, Kim S, Piccini JP, Fonarow GC, Lopes RD, Thomas L, et al. Use and associated risks of concomitant aspirin therapy with oral anticoagulation in patients with atrial fibrillation: insights from the Outcomes Registry for Better Informed Treatment of Atrial Fibrillation (ORBIT-AF) Registry. Circulation. 2013 ; 128: 721-728.

[20] Shah R, Hellkamp A, Lokhnygina Y, Becker RC, Berkowitz SD, Breithardt G, et al. Use of concomitant aspirin in patients with atrial fibrillation: Findings from the ROCKET AF trial. American Heart Journal. 2016; 179: 77-86.

[21] Alexander JH, Lopes RD, Thomas L, Alings M, Atar D, Aylward $\mathrm{P}$, et al. Apixaban vs. warfarin with concomitant aspirin in patients with atrial fibrillation: insights from the ARISTOTLE trial. European Heart Journal. 2014; 35: 224-232.

[22] Dans AL, Connolly SJ, Wallentin L, Yang S, Nakamya J, Brueckmann M, et al. Concomitant use of antiplatelet therapy with dabigatran or warfarin in the Randomized Evaluation of Long-Term Anticoagulation Therapy (RE-LY) trial. Circulation. 2013; 127: 634-640.

[23] Xu H, Ruff CT, Giugliano RP, Murphy SA, Nordio F, Patel I, et $a l$. Concomitant Use of Single Antiplatelet Therapy with Edoxaban or Warfarin in Patients with Atrial Fibrillation: Analysis from the ENGAGE AF-TIMI48 Trial. Journal of the American Heart Association. 2016; 5: e002587.

[24] Elvira Ruiz G, Caro Martínez C, Flores Blanco PJ, Cerezo Manchado JJ, Albendín Iglesias H, Lova Navarro A, et al. Effect of concomitant antiplatelet therapy in patients with nonvalvular atrial fibrillation initiating non $\square$ vitamin $\mathrm{K}$ antagonists. European Journal of Clinical Investigation. 2019; 49: e13161.

[25] Fox KAA, Velentgas P, Camm AJ, Bassand JP, Fitzmaurice DA, Gersh BJ, et al. Outcomes Associated with Oral Anticoagulants Plus Antiplatelets in Patients with Newly Diagnosed Atrial Fibrillation. JAMA Network Open. 2020; 3: e200107.

[26] Kumar S, Danik SB, Altman RK, Barrett CD, Lip GYH, Chatterjee $\mathrm{S}$, et al. Non-Vitamin $\mathrm{K}$ Antagonist Oral Anticoagulants and Antiplatelet Therapy for Stroke Prevention in Patients with Atrial Fibrillation: a Meta-Analysis of Randomized Controlled 
Trials. Cardiology in Review. 2016; 24: 218-223.

[27] Antithrombotic Trialists' (ATT) Collaboration, Baigent C, Blackwell L, Collins R, Emberson J, Godwin J, Peto R, et al. Aspirin in the primary and secondary prevention of vascular disease: collaborative meta-analysis of individual participant data from randomised trials. The Lancet. 2009; 373: 1849-1860.

[28] Baigent C, Collins R, Appleby P, Parish S, Sleight P, Peto R. ISIS-2: 10 year survival among patients with suspected acute myocardial infarction in randomised comparison of intravenous streptokinase, oral aspirin, both, or neither. The ISIS-2 (second International Study of Infarct Survival) Collaborative Group. British Medical Journal. 1998; 16: 1337-1343.

[29] Yasuda S, Kaikita K, Akao M, Ako J, Matoba T, Nakamura M, et al. Antithrombotic Therapy for Atrial Fibrillation with Stable Coronary Disease. New England Journal of Medicine. 2019; 381: 1103-1113.

[30] Saito Y, Kobayashi Y, Tanabe K, Ikari Y. Antithrombotic therapy after percutaneous coronary intervention from the Japanese perspective. Cardiovascular Intervention and Therapeutics. 2020; 35: 19-29.

[31] Yao X, Shah ND, Sangaralingham LR, Gersh BJ, Noseworthy
PA. Non-Vitamin K Antagonist Oral Anticoagulant Dosing in Patients with Atrial Fibrillation and Renal Dysfunction. Journal of the American College of Cardiology. 2017; 69: 2779-2790.

[32] Yamashita Y, Uozumi R, Hamatani Y, Esato M, Chun Y, Tsuji $\mathrm{H}$, et al. Current Status and Outcomes of Direct Oral Anticoagulant Use in Real-World Atrial Fibrillation Patients - Fushimi AF Registry. Circulation Journal. 2017; 81: 1278-1285.

[33] Steinberg BA, Shrader P, Pieper K, Thomas L, Allen LA, Ansell $\mathrm{J}$, et al. Frequency and Outcomes of Reduced Dose Non-Vitamin K Antagonist Anticoagulants: Results from ORBIT-AF II (the Outcomes Registry for Better Informed Treatment of Atrial Fibrillation II). Journal of the American Heart Association. 2018; 7: e007633.

[34] Cho MS, Yun JE, Park JJ, Kim YJ, Lee J, Kim H, et al. Outcomes after Use of Standard- and Low-Dose Non-Vitamin K Oral Anticoagulants in Asian Patients with Atrial Fibrillation. Stroke. 2018; STROKEAHA118023093.

[35] Lee S, Lee YS, Park J, Cha M, Kim T, Park J, et al. Label Adherence for Non-Vitamin K Antagonist Oral Anticoagulants in a Prospective Cohort of Asian Patients with Atrial Fibrillation. Yonsei Medical Journal. 2019; 60: 277-284. 